\title{
O uso de uma plataforma virtual para mobilização social no enfrentamento de arboviroses e controle do Aedes aegypti
}

\section{The use of a virtual platform for social mobilization in the confrontation of arboviruses and control of Aedes aegypti}

\section{El uso de una plataforma virtual para la movilización social para combatir los arbovirus y el control de Aedes aegypti}

Danielle Costa Silveira ${ }^{1, a}$

danielle.silveira@fiocruz.br | https://orcid.org/o00o-0001-9029-4257

Rose Ferraz Carmo ${ }^{2, b}$

rferrazcarmo@gmail.com | https://orcid.org/0000-0001-9703-8547

Fernanda Carvalho de Menezes ${ }^{1, c}$

menezes.bhe@hotmail.com | https://orcid.org/0000-0002-2413-1633

Juliana Ferreira de Oliveira ${ }^{1, b}$

jufer.oliveira@gmail.com | http://orcid.org/0000-0003-1585-0684

Nádia Ladendorff de Oliveira ${ }^{1, d}$

nadia.ladendorff@gmail.com | https://orcid.org/0000-0002-1191-2297

Vitor Davis Teixeira Aranha ${ }^{1, e}$

vitor.aranha@fiocruz.br | https://orcid.org/o0o0-0002-5329-9304

Zélia Maria Profeta da Luz ${ }^{1, f}$

zelia.profeta@fiocruz.br | https://orcid.org/o0o0-0002-0819-3025

${ }^{1}$ Fundação Oswaldo Cruz, Instituto René Rachou. Belo Horizonte, MG, Brasil.

${ }^{2}$ Escola de Saúde Pública de Minas Gerais. Belo Horizonte, MG, Brasil.

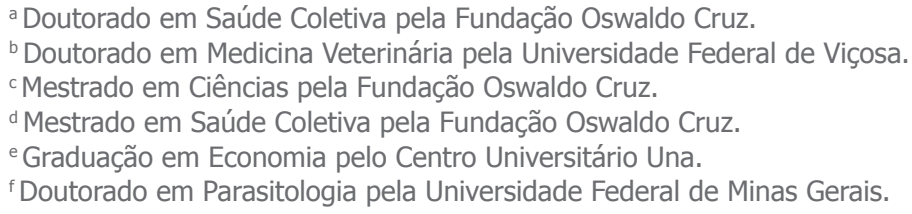

\section{RESUMO}

A mobilização social para o enfrentamento da dengue, zika, chikungunya e controle do Aedes aegypti tem sido um desafio no país. Esse artigo apresenta o processo de elaboração de uma plataforma virtual, incluindo a composição do seu acervo e a análise exploratória do seu uso, como ferramenta, numa proposta de mobilização social que envolve a criação de comitês populares nos territórios para o enfrentamento das três doenças e vetor. A construção da plataforma considerou: facilidade para usuário; acesso; armazenamento de material; interatividade e banco para monitoramento de dados. A seleção do acervo baseou-se em critérios: técnico, referente à fonte e autoria; acurácia, concordância entre informação e evidência em saúde; e legibilidade, garantindo a compreensão por diferentes grupos. A análise exploratória utilizou o banco de dados da plataforma. Os resultados apontaram a plataforma como uma ferramenta que pode contribuir com a comunicação e informação em saúde nessa proposta de mobilização social.

Palavras-chave: Arboviroses; Comunicação em Saúde; Mídias Sociais; Mobilização Social; Promoção da Saúde. 


\section{ABSTRACT}

Social mobilization for dengue, zika and chikungunya prevention and control of Aedes aegypti has been a challenge in the country. This paper presents the process of elaboration of a virtual platform, including the composition of its collection and the exploratory analysis of its use, as a tool in a social mobilization proposal that encompasses the creation of popular committees in the territories for the confrontation of the three diseases and vector. The construction of the platform considered: ease of use; access; material storage; interactivity and database for data monitoring. The selection of the collection was based on the following criteria: technical, considering source and authorship; accuracy, regarding the agreement between health information and evidence; legibility, ensuring the understanding by different groups. The exploratory analysis used the platform database. The results showed the platform as a tool which can contribute to health communication and information in this proposal of social mobilization.

Keywords: Arbovirus Infections; Health Communication; Social Media; Community Participation; Health Promotion.

\section{RESUMEN}

La movilización social para combatir el dengue, zika, chikungunya y control de Aedes ha sido un desafío. Este artículo presenta el proceso de elaboración de una plataforma virtual, que incluye la composición de su acervo y el análisis exploratorio de su uso, como herramienta en una propuesta de movilización social que implica la creación de comités populares en los territorios para enfrentar las enfermedades y el vector. La construcción de la plataforma consideró: facilidad para usuario; acceso; almacenamiento de material; interactividad y bases de datos para el monitoreo. La selección del acervo se basó en criterios: técnico, con fuente y autoría; precisión, concordancia entre información y evidencia en salud; legibilidad, para garantizar la comprensión por diferentes grupos. El análisis utilizó datos de la plataforma. Los resultados mostraron que la plataforma puede ser una herramienta para contribuir a la comunicación e información de salud en esta propuesta de movilización social.

Palabras clave: Infecciones por Arbovirus; Comunicación en Salud; Medios de Comunicación Sociales; Participación de la Comunidad; Promoción de la Salud.

\footnotetext{
Contribuição dos autores:

Silveira DC trabalhou na aquisição, análise e interpretação de dados e redação do manuscrito.

Menezes FC, Oliveira JF, Oliveira NL e Aranha VDT trabalharam na aquisição, análise e interpretação dos dados.

Carmo RF e Luz ZMP trabalharam na concepção e delineamento do estudo e na revisão crítica de conteúdo intelectual.

Declaração de conflitos de interesse: os autores declaram não haver conflitos de interesse.

Fontes de Financiamento: Fundação de Amparo à Pesquisa do Estado de Minas Gerais (FAPEMIG), Fundação Oswaldo Cruz (Fiocruz).
}

Considerações éticas: o estudo foi aprovado pelo Comitê de Ética e Pesquisa da Fiocruz Minas (Parecer 2.402.946).

Agradecimentos/Contribuições: à equipe da Fiocruz Minas, envolvida na pesquisa, à Secretaria de Estado de Educação e Secretaria de Estado de Saúde de Minas Gerais e à Escola de Saúde Pública de Minas Gerais.

Histórico do artigo: submetido: 27 jun. 2020 | aceito: 15 set. 2020. | publicado: 22 mar. 2021.

Apresentação anterior: não houve.

Licença CC BY-NC atribuição não comercial. Com essa licença é permitido acessar, baixar (download), copiar, imprimir, compartilhar, reutilizar e distribuir os artigos, desde que para uso não comercial e com a citação da fonte, conferindo os devidos créditos de autoria e menção à Reciis. Nesses casos, nenhuma permissão é necessária por parte dos autores ou dos editores. 


\section{INTRODUÇÃO}

A mobilização social para o enfrentamento das arboviroses dengue, zika e chikungunya e controle do Aedes aegypti tem sido um desafio no país. Fatores como a verticalidade do Programa de Controle, foco no vetor e culpabilização do morador têm contribuído para o baixo engajamento da população. No modelo de controle atual é destinado à população o papel de mera espectadora nas ações realizadas pelos profissionais de saúde (CLARO; TOMASSINI; ROSA, 2004; RANGEL-S, 2008; SILVA; MALLMANN; VASCONCELOS, 2015).

Dessa forma, na tentativa de contribuir com uma abordagem na qual a população atue como protagonista no controle das três doenças e do seu vetor, foi elaborada uma Proposta de Vigilância Comunitária em Saúde que visa à criação de comitês populares, localizados em escolas da rede pública estadual de ensino de Minas Gerais, compostos por alunos, pais, professores e membros da comunidade, que têm como tarefa atuar na perspectiva do território - entendido como espaço de produção e circulação de modos de vida e saúde (MAFRA; CHAVES; LOWEN; CAMARGO, 2015; LIMA, 2016; FERNANDES et al., 2017) - realizando o diagnóstico local e o planejamento de ações de enfrentamento às três doenças e proliferação do vetor, contribuindo para a criação de ambientes favoráveis à saúde, qualidade de vida e bem-estar.

Os princípios da promoção da saúde, empoderamento e participação social são entendidos como caminhos para fortalecer o protagonismo da população (SICOLI; NASCIMENTO, 2003; ARREAZA; MORAES, 2010; VASCONCELOS; OLIVEIRA-COSTA; MENDONÇA, 2016).

Também para a Organização Pan-Americana da Saúde (OPAS, 2004), a eficácia de iniciativas de promoção da saúde está relacionada à sua capacidade de induzir engajamento comunitário, estimulando a participação da população no processo de reconfiguração do território, o qual ocupa um lugar fundamental nas ações promotoras de saúde e de equidade (MAFRA; CHAVES; LOWEN; CAMARGO, 2015; LIMA, 2016; FERNANDES et al., 2017).

Buscando atender à necessidade de comunicação, troca de conhecimento e informação, para o desenvolvimento da Proposta de Vigilância Comunitária em Saúde, uma plataforma virtual foi criada como forma de facilitar os processos de organização dos comitês e de acompanhamento do trabalho nos territórios.

No presente artigo apresentamos o processo de elaboração da plataforma virtual inserida na Proposta de Vigilância Comunitária em Saúde, incluindo a composição do seu acervo e a análise exploratória do seu uso.

\section{MÉTODOS}

Trata-se de um estudo descritivo e exploratório que foi desenvolvido em duas etapas. A primeira correspondeu à descrição do processo de elaboração da plataforma virtual, incluindo a composição do seu acervo. Na segunda etapa foi realizada uma análise exploratória do uso da ferramenta pelos comitês populares participantes da Proposta.

\section{Etapa 1 - descrição do processo de elaboração da plataforma virtual e da composição do seu acervo}

O processo de elaboração da plataforma foi idealizado a partir de questões operacionais e de um processo de avaliação da qualidade da informação para a composição de seu acervo.

Como questões operacionais, a plataforma, caracterizada como uma mídia social (REDE MOBILIZADORES, 2016), foi planejada e construída de forma a proporcionar: 1) facilidade para usuários de diversos níveis de conhecimento na área de informática; 2) garantia de acesso por diferentes tipos/ velocidades de internet; 3) capacidade de armazenamento de material educativo em diferentes formatos; 
4) interatividade, a partir de fóruns de discussão, com possibilidade para compartilhamento de documentos e imagens; 5) geração de banco de dados para monitoramento de acessos.

A plataforma foi desenvolvida por um integrante da equipe de pesquisa, com o suporte técnico da informática do Instituto René Rachou, que é a Fiocruz em Minas Gerais. O monitoramento das configurações e atualizações da plataforma, bem como sua manutenção e hospedagem, está a cargo da instituição.

No que se refere à interatividade, a plataforma contava com um grupo de cinco tutores - estudantes de pós-graduação da Fiocruz Minas. Todos faziam parte da equipe de pesquisa e tinham como função acompanhar a constituição dos comitês populares e seu processo formativo. A tutoria também deveria atuar na comunicação e informação sobre os objetivos da Proposta, ações a serem realizadas e organização do processo de trabalho nos territórios.

Ainda referente à primeira etapa do estudo, a composição do acervo da plataforma virtual incluiu: definição dos temas que seriam inseridos na ferramenta pelos profissionais responsáveis pelo seu desenvolvimento; e levantamento e análise da qualidade da informação dos materiais de acordo com os temas previamente estabelecidos.

A definição dos temas foi baseada na identificação daqueles que fossem capazes de contemplar as diversas abordagens previstas para a formação e atuação dos comitês: 'doenças e vetor' (descrição, sinais e sintomas, características do vetor, formas de proliferação e de eliminação); conceito de 'território' e sua influência no processo saúde-doença; 'diagnóstico do território' - condições de vida e situação de saúde, considerando as características, problemas e potencialidades do território para a saúde da população e para a proliferação do vetor responsável pela transmissão das doenças - incluindo técnicas e instrumentos para sua realização; elaboração de 'planos de ação'; e importância da 'participação popular' na implementação de ações locais.

A seleção dos materiais foi iniciada pelo tema relacionado às doenças e ao vetor. Para isso, foi realizado um levantamento on-line utilizando-se como termos de busca: 'dengue', 'chikungunya', 'zika' e 'Aedes aegypti'. A pesquisa foi realizada nos sites da Fiocruz, Ministério da Saúde, Secretaria do Estado de Saúde de Minas Gerais e Fundo de População das Nações Unidas. Foram selecionados materiais educativos diversos, tais como vídeos, cartilhas, materiais infográficos, jogos, videoaulas, debates e entrevistas. O material selecionado frequentemente recomendava o acesso a outros materiais, principalmente no caso de vídeos, direcionando a pesquisa para o site de compartilhamento de vídeos Youtube.

Dos 53 materiais levantados, 35 (66\%) foram identificados como materiais educativos, ou seja, cujo conteúdo e formato de apresentação contribuíssem no processo de aprendizagem e discussão dos participantes (BANDEIRA, 2018). O restante não foi incluído na análise por se tratar de manuais técnicos e livros, cujo conteúdo poderia não ser compreendido por todos os participantes dos comitês populares, visto serem pessoas de diferentes idades e níveis de escolaridade. Reportagens e divulgação de curso on-line também compuseram o montante de materiais excluídos.

A análise dos materiais que comporiam o acervo da plataforma virtual foi baseada na avaliação da qualidade da informação adaptada da proposta metodológica desenvolvida pelo Laboratório Internet Saúde e Sociedade (LAISS) da Escola Nacional de Saúde Pública da Fundação Oswaldo Cruz (ENSP/Fiocruz) para avaliação da informação sobre dengue disponibilizada em sites da internet. O grupo propõe a adoção de cinco critérios para avaliação: técnico, acurácia, legibilidade, interatividade e abrangência (PEREIRA NETO; PAOLUCCI, 2014).

A opção por usar essa metodologia foi fundamentada pela adequação entre o objeto de investigação a que ela foi aplicada - avaliação da qualidade da informação em sites sobre dengue (PEREIRA NETO; PAOLUCCI, 2014) - e o objetivo do presente estudo: avaliar materiais educativos sobre dengue, chikungunya e zika para composição do acervo da plataforma virtual. Ainda que o objeto de investigação do grupo do LAISS tenha sido sites, entende-se que os critérios técnico, acurácia e legibilidade poderiam ser adaptados para avaliação de outros veículos de informação, tais como materiais impressos e audiovisuais. 
O quadro 1 apresenta a definição dos critérios técnico, acurácia e legibilidade, utilizados pelo grupo LAISS, e a forma como foram empregados/adaptados para seleção do acervo da plataforma virtual da Proposta de Vigilância Comunitária em Saúde.

\section{Quadro 1 - Critérios para avaliação da qualidade da informação utilizados na etapa de seleção do acervo da plataforma virtual, julho de 2016}

\begin{tabular}{|l|l|l|}
\hline Critério & Utilização pelo grupo LAISs & Aplicação no estudo \\
\hline Técnico & $\begin{array}{l}\text { Maneira como a informação foi } \\
\text { disponibilizada, incluindo a responsabilidade } \\
\text { pela informação oferecida, a existência de } \\
\text { datas de criação e de atualização do material, } \\
\text { existência de propagandas. }\end{array}$ & $\begin{array}{l}\text { Inclusão no acervo apenas de materiais } \\
\text { que apresentassem informações sobre a } \\
\text { fonte, autoria ou de responsabilidade pelas } \\
\text { informações. }\end{array}$ \\
\hline Acurácia & $\begin{array}{l}\text { Grau de concordância entre a informação } \\
\text { oferecida e a melhor evidência ou prática } \\
\text { em saúde geralmente aceita. Outros } \\
\text { termos também podem ser aplicados, como } \\
\text { "confiabilidade" ou "convencionalidade da } \\
\text { informação. }\end{array}$ & $\begin{array}{l}\text { Adaptado para o estudo, permitindo também a } \\
\text { análise da "atualidade da informação", devido } \\
\text { ao fato de zika e chikungunya representarem } \\
\text { discussões mais recentes e que, portanto, não } \\
\text { eram contempladas em materiais mais antigos. }\end{array}$ \\
\hline Legibilidade & $\begin{array}{l}\text { Capacidade de compreensão da informação } \\
\text { por diferentes grupos: profissionais de saúde, } \\
\text { profissionais de outras áreas, estudantes, } \\
\text { pessoas com pouca educação formal, pessoas } \\
\text { diretamente afetadas/envolvidas pelo assunto }\end{array}$ & $\begin{array}{l}\text { Adaptado para o estudo, tendo como referência } \\
\text { o perfil dos comitês populares, levando à } \\
\text { observação de questões como: linguagem } \\
\text { utilizada, clareza da informação e tempo de } \\
\text { duração, no caso de vídeo. }\end{array}$ \\
\hline
\end{tabular}

Fonte: adaptado de Pereira Neto e Paolucci (2014).

\section{Etapa 2 - análise exploratória do uso da ferramenta pelos comitês populares participantes da Proposta}

Na segunda etapa do estudo, a análise exploratória do uso da plataforma pelos comitês populares buscou analisar sua potencialidade enquanto ferramenta de comunicação e informação em saúde, bem como de apoio ao processo de mobilização social da Proposta.

À época do estudo, a Proposta contava com 49 comitês populares, formados por pessoas da comunidade escolar e moradores locais, envolvendo 39 municípios mineiros, de acordo com o quadro abaixo.

Quadro 2 - Comitês populares cadastrados na plataforma virtual da Proposta de Vigilância Comunitária em Saúde, de acordo com o município de localização da escola, janeiro de 2018

\begin{tabular}{|l|c|}
\hline \multicolumn{1}{|c|}{ Município } & $\begin{array}{c}\text { Comitês por } \\
\text { município }\end{array}$ \\
\hline Ponte Nova, Viçosa & 3 \\
\hline Divinópolis, Pará de Minas, Pitangui, Raul Soares, São João do Paraíso, Uberlândia & 2 \\
\hline \begin{tabular}{l} 
Andrelândia, Belo Horizonte, Bom Despacho, Canaã, Capitão Enéas, Cedro do Abaeté, Dom \\
Silvério, Francisco Dumont, Ibirité, Iguatama, Ipatinga, Itatiaiuçu, Itaúna, Lamim, Leandro \\
$\begin{array}{l}\text { Ferreira, Lima Duarte, Ouro Branco, Pains, Perdigão, Piracema, Piranga, Quartel Geral, } \\
\text { Queluzito, Ribeirão das Neves, Rio Espera, Santa Luzia, Santa Rita do Ibitipoca, Santana do } \\
\text { Paraíso, Sem Peixe, São Miguel do Anta, São Sebastião do Oeste }\end{array}$ \\
\hline Total
\end{tabular} & 1 \\
\hline
\end{tabular}

Fonte: elaboração dos autores, a partir da plataforma virtual. 
A análise exploratória compreendeu o ano de 2018 e utilizou dados gerados pela própria plataforma, o que permitiu coletar e registrar: 1) número de pessoas cadastradas nos comitês populares; 2) número de comitês formados; 3) perfil dos integrantes dos comitês, de acordo com sexo, faixa etária, escolaridade e tipo de vínculo com a escola; 4) número de acessos à plataforma; 5) comunicação, a partir do quantitativo e do conteúdo das mensagens trocadas entre comitês e tutores.

Para garantir o anonimato, os comitês estão identificados pela sigla CT, seguida de um número que os designa.

\section{RESULTADOS}

\section{A plataforma virtual da Proposta de Vigilância Comunitária em Saúde}

A plataforma foi moldada a partir de um layout que abarcava uma série de tópicos, como: apresentação e objetivos do projeto; relação das instituições envolvidas (Fiocruz Minas, Secretaria de Estado de Educação, Secretaria de Estado de Saúde e Escola de Saúde Pública de Minas Gerais); contextualização da tríplice epidemia como um problema de saúde pública; importância do envolvimento da população, por meio da criação de comitês populares; orientações e conteúdo formativo para atuação do comitê no território; espaço para interação e diálogo entre tutores e participantes da Proposta. Por meio desses conteúdos, a ideia era permitir um entendimento ampliado sobre a Proposta.

$\mathrm{O}$ acesso à plataforma era exclusivo às escolas participantes da Proposta de Vigilância Comunitária em Saúde, com o objetivo principal de possibilitar o processo formativo dos comitês, estimulando sua atuação no território por meio de ações coletivas e estratégias participativas que permitissem a elaboração de um diagnóstico da situação de saúde e das condições de vida, com vistas à criação de ambientes favoráveis à saúde e controle do Aedes aegypti.

\section{A composição do acervo da plataforma}

No que diz respeito à avaliação da informação utilizada nos materiais, os critérios técnico, acurácia e legibilidade contribuíram para a seleção final do conteúdo relacionado às doenças e ao vetor, com validação de 15 materiais, dos quais, 11 foram produzidos pela Fiocruz, três pelo Fundo de População das Nações Unidas e um pelo Ministério da Saúde. O quadro a seguir apresenta os materiais educativos selecionados e incluídos na plataforma. 
Quadro 3 - Materiais educativos relacionados às 'doenças e ao vetor', avaliados a partir dos critérios técnico, acurácia e legibilidade, selecionados e incluídos na plataforma virtual da Proposta de Vigilância Comunitária em Saúde, junho de 2016

\begin{tabular}{|c|c|c|}
\hline Tipo de material & Título & $\begin{array}{l}\text { Atendeu aos critérios } \\
\text { técnico, acurácia, } \\
\text { legibilidade? }\end{array}$ \\
\hline \multirow{7}{*}{$\begin{array}{l}\text { Conteúdo escrito } \\
\text { em site }\end{array}$} & Dengue & Sim \\
\hline & Chikungunya & Sim \\
\hline & Zika & Sim \\
\hline & Dengue, zika, chikungunya: entenda as diferenças & Sim \\
\hline & Dengue & Sim \\
\hline & Dengue: vírus e vetor & Sim \\
\hline & Histórias, direitos e zika & Sim \\
\hline Documentário & Mulheres do zika & Sim \\
\hline $\begin{array}{l}\text { Entrevista escrita } \\
\text { em site }\end{array}$ & $\begin{array}{l}\text { 'Ao que tudo indica, problema do zika está só começando; } \\
\text { temos muita dor pela frente' }\end{array}$ & Sim \\
\hline \multirow{4}{*}{ Vídeo } & $\begin{array}{l}\text { Aedes aegypti - Introdução aos Aspectos Científicos do } \\
\text { Vetor }\end{array}$ & Sim \\
\hline & $\begin{array}{l}\text { Aedes aegypti e Aedes albopictus: uma Ameaça aos } \\
\text { Trópicos }\end{array}$ & Sim \\
\hline & Ligado em Saúde: Dengue & Sim \\
\hline & Aedes aegypti: que mosquito é esse? & Sim \\
\hline Videoclipe & Mais direitos, menos zika & Sim \\
\hline $\begin{array}{l}\text { Reportagem escrita } \\
\text { em site }\end{array}$ & Doenças virais: seminário debate diagnóstico e atenção & $\operatorname{Sim}$ \\
\hline
\end{tabular}

Fonte: elaboração dos autores.

A utilização dos critérios técnico, acurácia e legibilidade mostrou-se uma alternativa apropriada para a seleção dos materiais para a plataforma virtual, uma vez que permitiu a inclusão de um quantitativo que não representasse um volume muito extenso, tampouco informações de caráter complexo, já que o perfil dos envolvidos nos comitês apontou a necessidade de conteúdos objetivos, sucintos e complementares essas questões orientaram a escolha dentre uma vasta opção de materiais disponíveis on-line.

O material sobre dengue, zika e chikungunya, do site da Fiocruz reunia informações de maneira clara e sucinta sobre várias questões que seriam importantes para os comitês, como características e formas de reprodução do vetor das doenças, panorama dos casos no país, sinais e sintomas, diagnóstico, formas de prevenção e tratamento.

Da mesma forma, o material 'Dengue, zika, chikungunya: entenda as diferenças' (Fiocruz) possuía informações que permitiam a compreensão das características comuns e específicas das doenças, principalmente no que se referia a sinais e sintomas e sobre a relação entre zika e casos de microcefalia.

O conteúdo sobre dengue no site do Ministério da Saúde apresentava uma discussão sobre a doença como um problema de saúde em vários países, além de um breve histórico no Brasil, problematizando a manutenção dos casos no país e os períodos de epidemia.

A série de vídeos 'Aedes aegypti - Introdução aos Aspectos Científicos do Vetor', também produzida pela Fiocruz, compreendia dez videoaulas, de curta duração cada, com imagem e áudio de boa qualidade. Com a participação de especialistas, o objetivo do material era abordar informações sobre o vetor de maneira mais informal, por meio de uma linguagem apropriada para diferentes públicos. Cada vídeo comentava sobre 
uma característica específica do vetor, com foco nas formas de impedir sua reprodução e manutenção no ambiente.

Todos esses materiais foram incluídos no processo formativo como conteúdo básico sobre 'doenças e o vetor'.

Os demais materiais descritos no quadro 3, apesar de atenderem igualmente aos critérios propostos, foram selecionados como conteúdo complementar na plataforma virtual, uma vez que foram percebidos como passíveis de contribuir de maneira mais adequada para aprofundar ou melhor explanar questões mais específicas que poderiam surgir nas discussões do comitê. Dessa forma, durante o acompanhamento do processo formativo dos comitês, os tutores da plataforma procuravam incentivar o acesso também a esses materiais, de acordo com as demandas e dúvidas apresentadas pelos participantes.

Os conteúdos referentes ao 'território', 'diagnóstico do território', 'plano de ação’ e 'participação popular' foram elaborados a partir de leituras, discussão e adaptação para o formato da plataforma virtual, possibilitando uma revisão do conhecimento já existente sobre o assunto e sua adaptação ao grupo, visando a contribuir para aumentar o interesse dos comitês sobre o problema e o significado da sua participação na pesquisa.

O fato desse conteúdo ter sido produzido exclusivamente para a discussão com os participantes da Proposta também permitiu uma maior articulação entre os temas, já que foram elaborados de maneira a promover uma interdependência entre os assuntos, procurando promover um conhecimento ampliado, capaz de lançar um novo olhar no enfrentamento à tríplice epidemia.

O material produzido pelo Programa de Formação de Agentes Locais de Vigilância em Saúde PROFORMAR (FIOCRUZ, 2004) também auxiliou na composição do acervo da plataforma, em especial no que tange à discussão sobre território e diagnóstico local. Elaborado para a formação profissional de agentes de saúde locais, o referido material procurava promover uma abordagem ampliada da determinação social, econômica e política dos processos de saúde e de doença a partir da conformação e transformação do território, entendido como espaço de vida, trabalho e de criação de laços sociais.

Após o levantamento, análise dos materiais e elaboração do conteúdo para os temas definidos como orientadores do processo formativo, a composição do acervo da plataforma foi finalizada, tornando-se disponível para os comitês populares constituídos no âmbito da Proposta de Vigilância Comunitária em Saúde.

\section{Comunicação e participação: análise do uso da plataforma da Proposta de Vigilância Comunitária em Saúde}

De acordo com o cadastro da plataforma, 411 pessoas estavam participando da Proposta. A faixa etária dos participantes variava entre seis e 67 anos, com média de 28 anos, e 70\% ( $n=288)$ eram do sexo feminino.

Do total, 364 (89\%) participantes possuíam vínculo direto com a escola à qual o comitê se vinculava (aluno, professor, função de direção ou funcionário de outros setores) e 47 (11\%) não tinham relação direta com a instituição (familiar de aluno, ex-aluno e outros). Esse dado está apresentado de maneira mais detalhada na figura abaixo. 


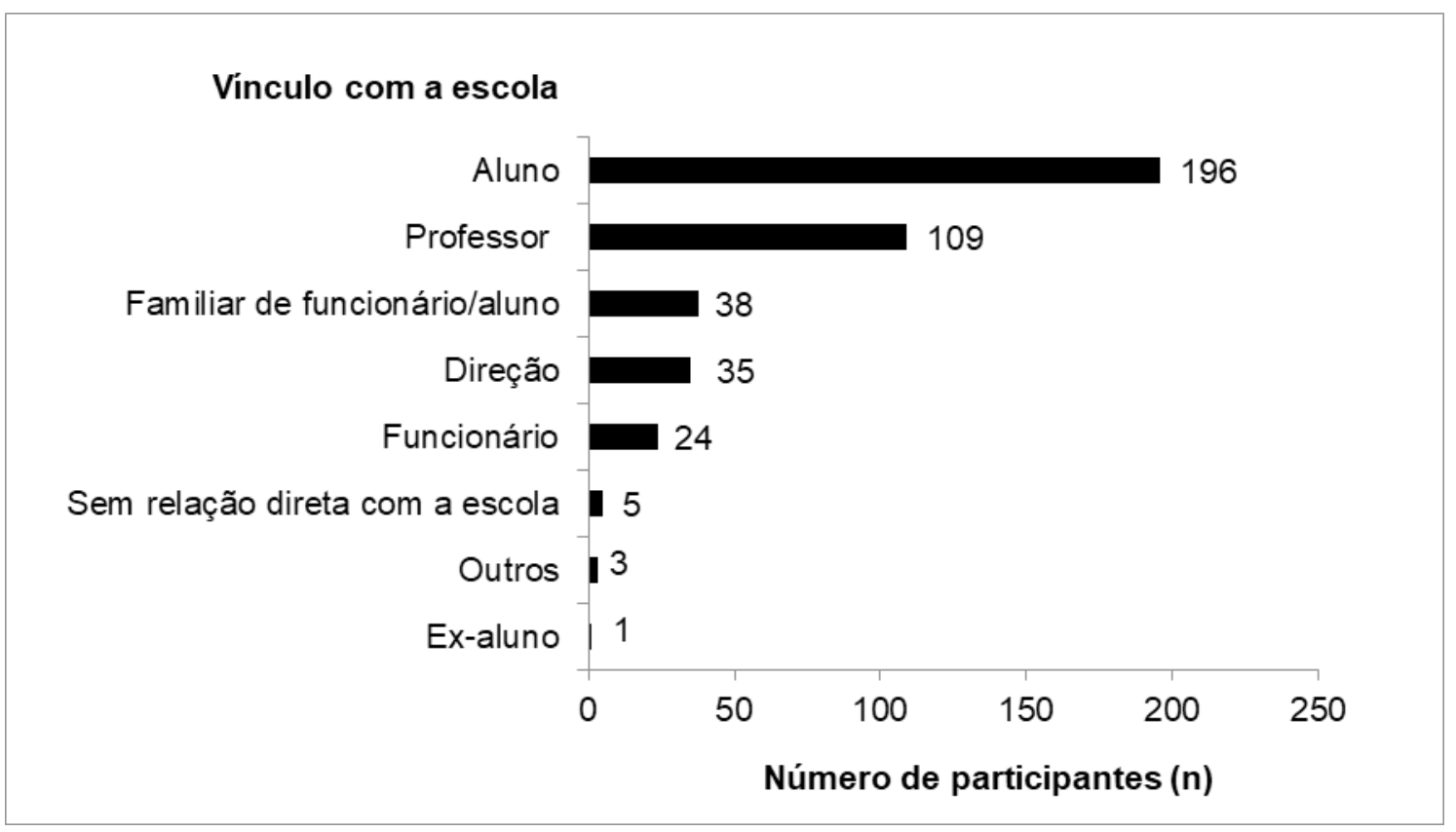

Figura 1 - Pessoas cadastradas na plataforma virtual da Proposta de Vigilância Comunitária em Saúde e sua relação com a escola, janeiro de 2018

Fonte: Plataforma virtual.

Em relação à escolaridade: 140 (34\%) possuíam ensino fundamental incompleto ou em curso; quatro (1\%), ensino fundamental completo; 74 (18\%), ensino médio incompleto ou em curso; 24 (6\%), ensino médio completo; quatro (1\%), ensino técnico profissionalizante; 136 (33\%), ensino superior completo ou em curso; e 29 (7\%) integrantes possuíam pós-graduação.

A análise do perfil dos participantes revelou um número expressivo de alunos ( $\mathrm{n}=196,48 \%)$, seguido pelos professores ( $n=109,27 \%)$. Familiar dos alunos $(n=38,9 \%)$ e membros da Direção das escolas ( $n=35$, 8\%) apareceram com números semelhantes (Figura 1).

Em relação à análise do processo de comunicação, os tutores, a partir de estratégias que denominaram 'chamadas aos comitês', estimularam o acesso, o diálogo e a troca de informação pela plataforma. Essas chamadas consistiam em mensagens elaboradas periodicamente pela equipe da pesquisa com o intuito de incentivar a comunicação com os participantes da Proposta e estimular o trabalho no território.

As chamadas alcançaram êxito em relação ao número de acessos na plataforma que, desde o início da Proposta, já foi visitada 3.196 vezes.

Em 2018, foram enviadas 74 mensagens pela plataforma. De maneira geral, os contatos entre tutores e comitês tinham o objetivo de esclarecer dúvidas sobre a Proposta em relação às etapas que precisavam ser cumpridas, atividades a serem realizadas, como encaminhá-las e prazos para a realização, conforme apresentado a seguir.

“O comitê pode ser formado só por professores?”(CT1).

"A proposta é de que o comitê seja formado de maneira mais ampliada, por integrantes com diferentes atuações no território, possibilitando um trabalho mais coletivo. Assim, além de professores, é importante pensar em um comitê onde haja espaço para alunos, familiares, moradores e trabalhadores locais, entre outros." (Tutores da plataforma on-line).

“Já realizamos o mapa falado e o Plano de Ação, qual será o próximo passo?”(CT2). 
"Após essas etapas concluídas e envio do material, é hora de desenvolver as ações planejadas no território. Estamos à disposição para o que precisarem” (Tutores da plataforma on-line).

A cada mensagem recebida, os tutores adotavam a prática de elaboração compartilhada do retorno à dúvida/demanda, contribuindo para que todas as questões fossem discutidas e decididas coletivamente entre os membros da equipe de pesquisa, qualificando a comunicação com os comitês e fortalecendo o desenvolvimento da Proposta no território, conforme exemplificado nos trechos a seguir.

“Vocês tiveram alguma dificuldade durante a realização do mapa falado? Como foi a experiência?” (Tutores da plataforma on-line).

O mapa falado foi realizado por todos os alunos da turma [...] A área foi escolhida no local onde fica a própria escola [...] A experiência foi muito gratificante, pois os alunos observaram e analisaram o próprio local onde vivem e pude perceber percepção ambiental deles com relação ao assunto" (CT 3).

"Como vai o plano de ação? O comitê está conseguindo desenvolver as atividades propostas? Foi necessário realizar alguma alteração no plano (atividades, recursos, cronograma)? Por favor, mande notícias de como anda o trabalho do comitê por aí" (Tutores da plataforma on-line).

"O Plano está sendo desenvolvido conforme cronograma. Estamos usando a criatividade e contando com patrocínio dos comerciantes locais quando se trata de alguma ação que demanda recurso financeiro. A prefeitura, através da Secretaria de Saúde, está sendo nossa parceira no sentido do mapeamento dos bairros e informações sobre as áreas de risco [...]” (CT 4).

\section{DISCUSSÃO}

A análise exploratória do uso da plataforma pelos comitês populares apontou resultados satisfatórios quanto à sua importância para promover comunicação, informação e participação na Proposta de Vigilância Comunitária em Saúde. Nesse sentido, tal ferramenta pode ser considerada uma alternativa para o desenvolvimento de práticas de intervenção e de vigilância comunitária em saúde, fortalecendo a mobilização social para o enfrentamento de problemas de grande magnitude e complexidade, como dengue, zika e chikungunya, que dependem de ações coordenadas que extrapolem o campo da saúde, envolvendo a população de forma ativa e permanente, tendo o território de vida e trabalho como cenário de atuação e transformação.

Também é importante ressaltar que optamos pela utilização de uma plataforma virtual na Proposta de mobilização para possibilitar, por meio da internet, a adesão do maior número possível de comitês em Minas Gerais, independentemente de sua localização no estado, que possui 853 municípios. Procuramos, ainda, promover articulação e acompanhamento contínuos no desenvolvimento das ações de mobilização nos territórios.

A respeito do uso da internet, esse tem sido um importante espaço para a promoção de ações participativas. Araújo, Penteado e Santos (2015), analisando processos de webativismo, afirmam que, por meio da internet, é possível ampliar e disseminar a voz do cidadão, desenvolvendo nossas formas de ativismo político. Pereira Júnior e Spitz (2016) corroboram, a partir de um estudo sobre tecnologias desenvolvidas pela academia, organizações não governamentais, associações comunitárias, entre outros, que tais setores contribuem para promover a participação e a inclusão da sociedade no desenvolvimento 
de iniciativas de empreendedorismo social. Miranda e Rocha (2018) analisaram o uso do Facebook como estratégia de comunicação para fortalecer a participação popular na gestão pública do setor saúde.

A internet é, portanto, uma estratégia consolidada de construção de redes e espaços coletivos para mobilização e ativismo. Por meio dela, pessoas se conhecem, descobrem afinidades e interesses em comum, e podem se articular para um propósito coletivo (ARAÚJO; PENTEADO; SANTOS, 2015; PEREIRA JÚNIOR; SPITZ, 2016; MIRANDA; ROCHA, 2018).

De acordo com dados do Instituto Brasileiro de Geografia e Estatística (IBGE), em 2017, 75\% da população brasileira já possuía acesso à internet no ambiente doméstico, sem contar nos demais espaços, como nas escolas públicas, local de acesso à plataforma virtual da Proposta de Vigilância Comunitária em Saúde (IBGE, 2017). Na análise do IBGE, o acesso à internet em áreas rurais também tem apresentado um crescimento importante, fato que pode contribuir para incentivar a constituição de comitês populares nessas localidades.

Recorremos, ainda, às palavras de Henriques (2005) para contribuir nessa discussão, que afirma que os espaços para mobilização e participação precisam evoluir e serem condizentes com o momento. Nesse sentido, o fato de vivermos em uma época fortemente marcada pelo uso da internet representa uma importante abertura ao uso de uma plataforma virtual como ferramenta de comunicação e informação em saúde para envolver a população no enfrentamento da dengue, zika, chikungunya e controle do Aedes aegypti.

Experiências exitosas na diminuição de casos e controle do vetor têm se fundamentado em propostas mais horizontais, que valorizem e incluam a população, apontando para resultados mais efetivos e duradouros das ações desenvolvidas. Sinalizam a importância de ampliar o diálogo com a população e, ainda, de considerar a realidade, o contexto e a pluralidade dos territórios (ÁVILA; MARTÍNEZ; SHERMAN; FERNÁNDEZ CERNA, 2004; SOMMERFELD; KROEGER, 2012).

Dessa forma, ao estimular a participação da população, por meio de comitês populares, a plataforma representou uma maneira de concretizar o discurso da promoção da saúde, promovendo a discussão da saúde enquanto um produto social e ressaltando a necessidade de ações coletivas que incidam sobre seus determinantes e que promovam saúde e qualidade de vida (SICOLI; NASCIMENTO, 2003; ARREAZA; MORAES, 2010; VASCONCELOS; OLIVEIRA-COSTA; MENDONÇA, 2016).

Quanto ao processo de composição do acervo da plataforma, orientamo-nos por autores que ressaltam a importância da informação qualificada para a comunicação em saúde (HENRIQUES, 2005; ARAÚJO; CARDOSO, 2007; EMERICH; CAVACA; GENTILLI; EMERICH, 2016).

Nesse sentido, a adoção do tema 'território' como um conceito transversal na Proposta teve o intuito de permitir a contextualização da tríplice epidemia como um problema de saúde relacionado e afetado por características e fatores que, de diferentes formas e proporções, determinam o lugar de vida - tal reflexão é fundamental para o fomento de ações de promoção da saúde (SICOLI; NASCIMENTO, 2003; ARREAZA; MORAES, 2010; VASCONCELOS; OLIVEIRA-COSTA; MENDONÇA, 2016). Ao evidenciar esse tema, pretendeu-se fomentar um olhar mais atento para as diversidades e singularidades do território - suas características ambientais, econômicas, políticas, sociais e culturais - contribuindo para a discussão do quanto podem influenciar na ocorrência da tríplice epidemia, bem como sobre a importância de ações e políticas públicas para a criação de ambientes saudáveis (CAPRARA; OLIVEIRA; PEIXOTO, 2013; LIMA; GOURLAT; ROLIM NETO, 2015).

$\mathrm{Na}$ abordagem das 'doenças e vetor', diversos trabalhos demonstram que o conteúdo e a qualidade das informações veiculadas nas campanhas têm se mostrado ineficazes, deixando de lado uma discussão ampliada do problema que permita maior compreensão pela população e o reconhecimento de atitudes e práticas capazes de se configurar como soluções alternativas de enfrentamento. Ainda nesse sentido, 
mesmo com o excesso de informações, como acontece geralmente em períodos de epidemia, o que pode inclusive provocar a banalização das doenças, é preciso pensar também na maneira como esse conteúdo é compartilhado com a população, já que a compreensão de uma determinada situação enquanto um problema pode favorecer a produção de novos significados e atitudes entre os envolvidos (CLARO; TOMASSINI; ROSA, 2004; RANGEL-S, 2008; SILVA; MALLMANN; VASCONCELOS, 2015).

A inclusão dos temas 'diagnóstico do território' e 'plano de ação' foi fundamental como forma de apontar possibilidades de práticas, estratégias, e formas de intervenção para o desenvolvimento de ações de promoção da saúde territorializadas.

Assim, procuramos fortalecer o conceito de que a comunicação e a informação em saúde precisam romper com a ideia de acesso diferenciado da população ao conhecimento, de forma que esse seja incorporado a suas práticas e modos de viver a vida (RANGEL-S, 2008; VASCONCELOS; OLIVEIRA-COSTA; MENDONÇA, 2016; HENRIQUES, 2005).

A incorporação de tutores na plataforma foi a maneira utilizada na Proposta para estabelecer diálogo, interação e troca com os comitês populares. Para isso, recorremos a autores que descrevem o importante papel desses atores em ambientes virtuais de aprendizagem, onde o tutor, orientado por diretrizes educativas, tecnológicas e comunicativas, deve assumir a função de acompanhamento, orientação e motivação para uma aprendizagem autônoma, fundamentada no diálogo, respeito, troca de conhecimento e abertura a novos saberes (SOUZA; SPANHOL; LIMAS; CASSOL, 2004).

A interação entre os tutores e os comitês influenciou na visita ao acervo da plataforma, elaborado como aporte para aquisição de novas informações e ampliação do conhecimento. Em processos de mobilização social, essa é uma importante forma de empoderamento dos sujeitos, permitindo-lhes uma participação ativa e consciente nas ações (HENRIQUES, 2005).

A comunicação entre esses atores também fomentou a discussão de estratégias que pudessem incentivar ainda mais a participação na Proposta. Em função disso, o grupo de pesquisa vem trabalhando atualmente em melhorias na plataforma, sobretudo no seu layout, procurando torná-lo mais atrativo e interativo, principalmente para o público mais jovem.

Como limitação, compreendemos que a inclusão de dados primários, como entrevistas com os participantes da plataforma virtual, poderia contribuir para compreender algumas especificidades. No entanto, esse não foi nosso objetivo e, por isso, não foi incluído nesse primeiro momento do estudo.

\section{CONCLUSÃO}

A plataforma virtual representou uma interessante alternativa de comunicação e informação em saúde para a participação e disseminação da Proposta de Vigilância Comunitária em Saúde, visando à mobilização social no enfrentamento da dengue, zika e chikungunya e controle do Aedes aegypti em Minas Gerais.

\section{REFERÊNCIAS}

ARAÚJO, Inesita Soares de; CARDOSO, Janine Miranda. Comunicação e Saúde. Rio de Janeiro: Editora Fiocruz, 2007.

ARAÚJO, Rafael de Paula Aguiar; PENTEADO, Cláudio Luís de Camargo; SANTOS, Marcelo Burgos Pimentel. Democracia digital e experiências de e-participação: webativismo e políticas públicas. História, Ciências, Saúde - Manguinhos, Rio de Janeiro, v. 22, supl., p.1597-1619, dez. 2015. DOI: http://dx.doi. org/10.1590/S0104-59702015000500004. Disponível em: https://www.scielo.br/scielo.php?script=sci arttext\&pid=S0104-59702015001001597\&lng=en\&nrm=iso\&tlng=pt. Acesso em 1 abr. 2018. 
ARREAZA, Antonio Luis Vicente; MORAES, José Cassio. Vigilância da saúde: fundamentos, interfaces e tendências. Ciência \& Saúde Coletiva, Rio de Janeiro, v. 15, n. 4, p. 2215-2228, jul. 2010. DOI: https:// doi.org/10.1590/S1413-81232010000400036. Disponível em: http://www.scielo.br/scielo.php?script=sci arttext\&pid=S1413-81232010000400036. Acesso em 30 mar. 2019.

ÁVILA MONTES, Gustavo Adolfo; MARTíNEZ, Mercedes; SHERMAN, Catalina; FERNÁNDEZ CERNA, Eduardo. Evaluación de un módulo escolar sobre dengue y Aedes aegypti dirigido a escolares em Honduras. Revista Panamericana de Salud Pública, Washington, DC, v. 16, n. 2, p. 84-94, 2004. Disponível em: https:// www.scielosp.org/article/rpsp/2004.v16n2/84-94/. Acesso em 30 mar. 2019.

BANDEIRA, Denise. Material didático: conceito, classificação geral e aspectos da elaboração. [S. I.; s. n., 2018]. Disponível em: http://www2.videolivraria.com.br/pdfs/24136.pdf. Acesso em: 10 set. 2018.

CAPRARA, Andrea; OLIVEIRA, José Wellington; PEIXOTO, Ana Carolina Rocha (Org.). Ecossaúde, uma abordagem eco-bio-social: percursos convergentes no controle do dengue. Fortaleza: EdUECE, 2013.

CLARO, Lenita Barreto; TOMASSINI, Hugo Coelho Barbosa; ROSA, Maria Luiza Garcia. Prevenção e controle do dengue: uma revisão de estudos sobre conhecimentos, crenças e práticas da população. Cadernos de Saúde Pública, Rio de Janeiro, v. 20, n. 6, p. 1447-1457, dez. 2004. DOI: https://doi.org/10.1590/ S0102-311X2004000600002. Disponível em: http://www.scielo.br/scielo.php?script=sci arttext\&pid=S0102311X2004000600002. Acesso em: 30 abr. 2016.

EMERICH, Tatiana Breder; CAVACA, Aline Guio; GENTILLI, Victor; EMERICH, Adauto. Necessidades de saúde e direito à comunicação em tempos de midiatização. Revista Eletrônica de Comunicação, Informação e Inovação em Saúde, Rio de Janeiro, v. 10, n. 4, 2016. DOI: https://doi.org/10.29397/reciis. v10i4.1065. Disponível em: https://www.reciis.icict.fiocruz.br/index.php/reciis/article/view/1065. Acesso em: 20 dez. 2019.

FERNANDES, Valcler Rangel et al. O lugar da vigilância no SUS: entre os saberes e as práticas de mobilização social. Ciência \& Saúde Coletiva, Rio de Janeiro, v. 22, n. 10, p. 3173-3181, out. 2017. DOI: https://doi.org/10.1590/1413-812320172210.1772017. Disponível em: https://www.scielo.br/scielo. php?script=sci_arttext\&pid=S1413-81232017021003173\&lng=en\&nrm=iso\&tlng=pt. Acesso em 30 mar. 2018.

FUNDAÇÃO OSWALDO CRUZ (FIOCRUZ). Escola Politécnica de Saúde Joaquim Venâncio (Org.). 0 território e a vigilância em saúde. Rio de Janeiro: PROFORMAR, 2004.

HENRIQUES, Márcio Simeone. Comunicação, comunidades e os desafios da mobilização social. In: CONGRESSO BRASILEIRO DE CIÊNCIAS DA COMUNICAÇÃO, 28., 5-9 set. 2005, Rio de Janeiro. Anais [...]. Rio de Janeiro: Universidade Estadual do Rio de Janeiro, 2005. Disponível em: https://prefeitura. pbh.gov.br/sites/default/files/estrutura-de-governo/cultura/6.\%20Comunica\%C3\%A7\%C3\%A30\%2C\%20 comunidades $\% 20$ e $\% 20$ os $\% 20$ desafios $\% 20$ da $\% 20$ mobiliza $\%$ C3\%A7\%C3\%A30\%20social\%20 (M\%C3\%A1rcio\%20Simeone).pdf. Acesso em 30 mar. 2019.

INSTITUTO BRASILEIRO DE GEOGRAFIA E ESTATÍSTICA (IBGE). Pesquisa Nacional por Amostra de Domicílios Contínua. Acesso à internet e à televisão e posse de telefone móvel para uso pessoal. Rio de Janeiro: IBGE, 2017. E-book. Disponível em: https://biblioteca.ibge.gov.br/visualizacao/livros/liv101631 informativo.pdf. Acesso em 5 jun. 2019.

LIMA, Estelita Pereira; GOULART, Marília Oliveira Fonseca; ROLIM NETO, Modesto Leite. Meta-analysis of studies on chemical, physical and biological agents in the control of Aedes aegypti. BMC Public Health, [s. I.], n.15, p.858, 2015. DOI: https://doi.org/10.1186/s12889-015-2199-y. Disponível em: https://bmcpublichealth. biomedcentral.com/track/pdf/10.1186/s12889-015-2199-y. Acesso em: 30 jan. 2018.

LIMA, Samuel do Carmo. Território e promoção da saúde: perspectivas para a atenção primária à saúde. Jundiaí: Paco Editorial, 2016.

MAFRA, Melissa dos Reis Pinto; CHAVES, Maria Marta Nolasco; LOWEN, Ingrid Margareth Voth; CAMARGO, Jaqueline. O território como base para a intervenção em saúde. Caminho Aberto - Revista de Extensão do IFSC, Florianópolis, ano 2, n. 2, p. 48-56, jan./jun. 2015. DOI: http://dx.doi.org/10.35700/ca.2015.ano2n2.p4856.1512. Disponível em: https://periodicos.ifsc.edu.br/index.php/caminhoaberto/article/view/1512. Acesso em: 20 dez. 2019.

MIRANDA, Fernanda Santana; ROCHA, Dais Gonçalves. O uso do Facebook na promoção da saúde: uma revisão bibliográfica sobre empoderamento e participação popular. Revista Eletrônica de Comunicação, Informação e Inovação em Saúde, Rio de Janeiro, v. 12, n. 2, p. 232-243, 2018. DOI: https://doi. 
org/10.29397/reciis.v12i2.1331. Disponível em: https://www.reciis.icict.fiocruz.br/index.php/reciis/article/ view/1331. Acesso em $20 \mathrm{dez} .2019$.

ORGANIZACIÓN PANAMERICANA DE LA SALUD (OPAS). División de Promoción y Protección de la Salud. Municipios y comunidades saludables: guía de los alcaldes para promover calidad de vida. Washington, DC: Opas, 2004. Disponível em: https://iris.paho.org/handle/10665.2/42570. Acesso em: 3 abr. 2017.

PEREIRA JÚNIOR, Clorisval; SPITZ, Rejane. Plataformas digitais para participação cívica: inclusão digital e inovação social digital. In: CONGRESSO BRASILEIRO DE PESQUISA E DESENVOLVIMENTO EM DESIGN, 16., 2016, Belo Horizonte. Anais [...], São Paulo, v. 2, n. 9, p. 3123-3133, 2016. Disponível em: https://www. proceedings.blucher.com.br/article-details/plataformas-digitais-para-participao-cvica-incluso-digital-e-inovaosocial-digital-24504. Acesso em 20 set. 2018.

PEREIRA NETO, André de Faria; PAOLUCCI, Rodolfo. Qualidade da informação em sites de dengue: análise de uma experiência inovadora. Rio de Janeiro: ENSP, 2014.

RANGEL-S, Maria Ligia. Dengue: educação, comunicação e mobilização na perspectiva do controle propostas inovadoras. Interface - Comunicação, Saúde, Educação, São Paulo, v. 12, n. 25, p. 433-441, abr./jun. 2008. DOI: https://doi.org/10.1590/S1414-32832008000200018. Disponível em: https://www.scielo.br/ scielo.php?script=sci arttext\&pid=S1414-32832008000200018\&lng=en\&nrm=iso\&tlng=pt. Acesso em: 7 out. 2017.

REDE MOBILIZADORES. Internet e redes sociais como ferramentas de mobilização: material de apoio à Oficina Redes Sociais e Mobilização. [S. I.]: Rede Mobilizadores, 2016. E-book. Disponível em: http://www. mobilizadores.org.br/wp-content/uploads/2016/01/Cartilha-Redes-Sociais-e-Mobilizacao.pdf. Acesso em: 4 set. 2018.

SÍCOLI, Juliana Lordello; NASCIMENTO, Paulo Roberto do. Promoção da Saúde: concepções, princípios e operacionalização. Interface - Comunicação, Saúde, Educação, São Paulo, v. 7, n. 12, p. 101-1022, fev. 2003. DOI: https://doi.org/10.1590/S1414-32832003000100008. Disponível em: http://www.scielo.br/scielo. php?pid=s1414-32832003000100008\&script=sci abstract\&tlng=pt. Acesso em 29 mar. 2019.

SILVA, Ivanise Brito; MALLMANN Danielli Gavião; VASCONCELOS, Eliane Maria Ribeiro. Estratégias de combate à dengue através da educação em saúde: uma revisão integrativa. Saúde, Santa Maria, v. 41, n.2, p. 27-34, jul./dez. 2015. DOI: https://doi.org/10.1590/S1414-32832008000200018. Disponível em: https:// periodicos.ufsm.br/revistasaude/article/view/10955/pdf_1. Acesso em: 5 maio 2016.

SOMMERFELD, Johannes; KROEGER, Axel. Eco-bio-social research on dengue in Asia: a multicountry study on ecosystem and community-based approaches for the control of dengue vectors in urban and periurban Asia. Pathogens and Global Health, London, v. 106, n. 8, p. 428-435, 2012. DOI: https://dx.doi. org/10.1179\%2F2047773212Y.0000000055. Disponível em: https://www.ncbi.nlm.nih.gov/pmc/articles/ PMC3541880/. Acesso em: 30 mar. 2019.

SOUZA, Carlos Alberto de; SPANHOL, Fernando José; LIMAS, Jeane Cristina de Oliveira; CASSOL, Marlei Pereira. Tutoria na educação a distância. In: CONGRESSO INTERNACIONAL DE EDUCAÇÃO A DISTÂNCIA, 11., 7-10 set. 2004, Salvador. Anais [...]. São Paulo: Abed, 2004. Disponível em: http://www.abed.org.br/ congresso2004/por/htm/088-TC-C2.htm. Acesso em 19 set. 2018.

VASCONCELOS, Wagner Robson Manso de; OLIVEIRA-COSTA, Mariella Silva; MENDONÇA, Ana Valéria Machado. Promoção ou prevenção? Análise das estratégias de comunicação do Ministério da Saúde no Brasil de 2006 a 2013. Revista Eletrônica de Comunicação, Informação e Inovação em Saúde, Rio de Janeiro, v. 10, n. 2, abr./jun. 2016. DOI: https://doi.org/10.29397/reciis.v10i2.1019. Disponível em: https://www.reciis.icict. fiocruz.br/index.php/reciis/article/view/1019. Acesso em 20 dez. 2019. 